\title{
Um Estudo sobre a Integração dos Níveis de Análise dos Sistemas de Valores
}

\author{
Cicero Pereira ${ }^{1}$ \\ Universidade Católica de Goiás \\ Leoncio Camino \\ Joseli Bastos da Costa \\ Universidade Federal da Paraíba
}

\begin{abstract}
Resumo
Este artigo apresenta uma investigação sobre a integração dos níveis de análise dos sistemas de valores de estudantes universitários. No estudo realizado ( $N=406$ ), foram identificados 4 sistemas subjacentes à organização dos valores dos estudantes: materialista, hedonista, religioso e pós-materialista. A análise da convergência entre os sistemas e os tipos motivacionais da teoria de Schwartz mostrou a semelhança entre o materialismo e os valores da autopromoção, entre o sistema hedonista e a abertura à mudança, entre o sistema religioso e os valores de conservação e entre o pós-materialismo e o tipo motivacional da autotranscendência. Na discussão, enfatiza-se a natureza societal dos valores relacionando suas fontes às ideologias sociais.

Palavras-chave: Níveis de análises; valores; sistemas de valores.
\end{abstract}

\section{A Study about the Integration of the Analysis Levels of the Value Systems}

\begin{abstract}
This work is a study on the articulation of the analysis levels of university students' value systems. The results ( $N=406)$ show 4 systems underlying the organization of the students' value systems: materialist; hedonist; religious; post-materialist. The analyses of the convergences between values systems and motivacional types of Schwartz's theory showed that there are correlations between materialist and self-enhancement values, between hedonist system and opening to change values types, between religious and conservation values, and between post-materialism and self-transcendence motivational value type. The discussion emphasizes the societal nature of the values system connecting their sources to social ideologies.

Keywords: Analysis levels; values; values systems.
\end{abstract}

Os valores sociais são fundamentais na organização dos sistemas de crenças dos indivíduos (Rokeach, 1979a). Eles servem como padrões ou critérios que orientam ações, escolhas, julgamentos, atitudes e explicações sociais (Rokeach, 1979b; Williams, 1979), estão entre as crenças avaliativas mais importantes (Feather, 1990; Seligman \& Katz, 1996) e ocupam uma posição central na rede cognitiva que fundamenta a organização das pessoas em sociedades (Rokeach, 1968). Além disso, são amplamente compartilhados pelos grupos sociais e sua validade raramente é questionada (Maio \& Olson, 1998; Maio, Olson, Allen \& Bernard, 2001). Entretanto, o conjunto de estudos sobre os valores é marcado por contradições geradas pela diversidade de abordagens teóricas que orientam as pesquisas (Torres \& cols., 2001). De fato, há várias teorias sobre os valores. Dentre elas destacam-se a tipologia dos valores proposta por Rokeach (1973), a teoria dos tipos motivacionais (Schwartz, 1992) e a teoria dos valores materialistas e pós-materialistas (Inglehart, 1977). Este artigo realiza uma análise dessas tipologias e apresenta uma nova abordagem sobre esse tema que procura integrar diversos níveis de análise dos sistemas de valores. Essa abordagem se faz a partir de uma análise societal dos valores, utilizando um instrumento para medida dos sistemas de valores que regem as sociedades.

\section{Valores: Natureza e Abordagens Teóricas}

Doise (1982) distingue quatro níveis de análises nas explicações elaboradas pelos psicólogos sociais para os fenômenos sociais:

${ }^{1}$ Endereço para correspondência: Grupo de Pesquisa em Comportamento Político, Caixa Postal 5069, Cidade Universitária, 58051 970, João Pessoa, PB. E-mail: cicero.psi@ucg.br intraindividual; interindividual; intergrupal; societal. Explicações intraindividuais são as que analisam os fenômenos sociais com base em motivações psicológicas do indivíduo. No nível interindividual, as explicações são baseadas nas relações que ocorrem entre um indivíduo e outro indivíduo em uma dada situação. No nível intergrupal, focaliza-se a dinâmica das relações que os grupos sociais mantém com os outros grupos, sendo os fenômenos sociais dependentes da identificação dos indivíduos com esses grupos (Doise, 1976). No nível societal, as explicações baseiam-se nas ideologias subjacentes à forma como os grupos sociais se relacionam (Camino, 1996).

As explicações que a Psicologia Social tem dado aos valores são intraindividuais (Torres \& cols., 2001). Rokeach (1979a), por exemplo, conceitua os valores como crenças que o indivíduo possui sobre as formas de comportamento ou sobre os estados finais de existência que são mais adequados em uma determinada situação. Nessa perspectiva, as fontes dos valores estão, sobretudo, nas necessidades individuais (Rokeach, 1973), as quais, distribuídas hierarquicamente, organizam as crenças dos indivíduos em um sistema de valores. Nessa definição, duas características dos valores são importantes à compreensão de sua natureza: os valores são crenças; eles se referem a modos de condutas ou estados finais de existência. A primeira característica diz respeito ao conhecimento sobre as formas de comportamento e as finalidades das ações (Rokeach, 1968). A segunda característica distingue os valores instrumentais, meios utilizados para alcançar as preferencias individuais, dos valores terminais, as próprias preferências. Com base nessas características, Rokeach (1973) apresenta uma escala - Rokeach's Value Survey (RVS) - contendo 18 valores instrumentais e 18 terminais, os quais os sujeitos deveriam hierarquizar como princípios guia em suas vidas. 
Embora tenha servido de base para a maioria das pesquisas sobre valores nos anos 1980 (Bond, 1988; Braithwaite \& Scott, 1991; Chinese Culture Connection, 1987), a perspectiva teórico-metodológica subjacente ao RVS tem sido questionada (Braithwaite, 1994; Braithwaite \& Law, 1985). Esse questionamento refere-se ao fato dos procedimentos metodológicos empregados não captarem as estratégias utilizadas pelos sujeitos para classificar os valores. Com isso, não é possível comprovar empiricamente um aspecto central da teoria: a distinção entre valores instrumentais e terminais (Schwartz \& Bilsky, 1990). Para superar essa limitação, Schwartz (1992) reformula a teoria de Rokeach (1973) e propõe uma tipologia universal das motivações subjacentes aos valores dos indivíduos. Nessa perspectiva, Schwartz e Bilsky (1987) articulam a desejabilidade social dos valores, indicada por Kluckhohn (1968), com as características da teoria de Rokeach (1973). Com base nessa unificação, Schwartz (1999) define os valores como concepções que o indivíduo possui sobre o desejável, as quais, por serem transituacionais, guiam os atores sociais na elaboração de seu comportamento e na avaliação das pessoas. Para Schwartz (1992), todos os valores que as pessoas possuem estão ao serviço de 10 tipos motivacionais: poder, realização, hedonismo, estimulação, autodireção, universalismo, benevolência, tradição, conformidade e segurança. Por representarem as motivações humanas, as fontes dos valores estão localizadas em três necessidades básicas e universais na natureza humana (Schwartz, 1994): biológicas, de interação social estável e de sobrevivência dos grupos.

Para avaliar os tipos motivacionais, Schwartz (1992) apresenta o Schwartz's Values Survey (SVS). Os resultados de uma Smallest Space Analysis (Canter, 1985; Davison, 1983; Guttman, 1968) aplicada aos dados obtidos com o SVS mostram, em duas dimensões bipolares, relações de compatibilidade e de conflito entre os tipos motivacionais: autotranscendência (universalismo e benevolência) versus autopromoção (poder e realização); abertura à mudança (autodireção, estimulação e hedonismo) versus conservação (segurança, tradição e conformidade). A primeira dimensão organiza-se em torno do conflito que o indivíduo sente entre lutar pelo bem-estar coletivo e buscar o sucesso pessoal. A segunda dimensão é organizada pelo conflito entre as necessidades de mudança e a necessidade de manutenção do statu quo. Embora essa tipologia tenha sido observada em diversas culturas (Schwartz \& Bardi, 2001), sua universalidade não tem sido verificada. Menezes e Campos (1997), por exemplo, constatam variações em função do desenvolvimento cognitivo dos indivíduos e, no Brasil, Tamayo e Schwartz (1993) identificam, numa amostra de professores do ensino médio, oito tipos de valores. Essa constatação indica a necessidade de articular a abordagem intraindividual dos valores, representadas nos trabalhos de Rokeach (1973) e de Schwartz (1992), com uma abordagem sociológica, como a proposta por Inglehart (1977) sobre as mudanças nos valores culturais.

Inglehart (1977) concebe os valores como indicadores de mudanças culturais. Para ele, as transformações ocorridas nas formas de produção das sociedades ocidentais pós-modernas se relacionam a modificações que ocorrem na hierarquia de valores dessas sociedades. As mudanças culturais, observadas na emergência de novos valores, acompanham, igualmente, as modificações nas condições de produção dessas sociedades. Inglehart (1991) chama esse processo de feedback econômico-cultural e descreve dois conjuntos de valores como seus indicadores: valores materialistas e pós-materialistas. Segundo este autor, as condições de produção do início do capitalismo viabilizaram a emergência dos valores materialistas e a prioridade dada a esses valores favoreceu ao desenvolvimento do capitalismo nas sociedades industriais. Além disso, Inglehart (1994) considera, com base nas teses de Weber (1904/ 1994) sobre o desenvolvimento do capitalismo, que os valores materialistas indicam a mudança dos valores religiosos do feudalismo para uma sociedade laica e materialista. Do mesmo modo, com a estabilidade sócio-econômica de algumas sociedades pós-industriais, tem-se verificado, nas últimas décadas, a emergência de metas pósmaterialistas cuja valorização favorece ao desenvolvimento econômico dessas sociedades.

Segundo Inglehart (1977), as sociedades com problemas sociais básicos, como a estabilidade econômica, priorizam os valores materialistas, enquanto as sociedades que solucionaram esses problemas valorizam metas pós-materialistas. Para testar essa hipótese, Inglehart (1991) apresenta um instrumento para medir os valores materialistas e pós-materialistas. Os indicadores materialistas avaliam a importância da segurança física e econômica, enquanto os indicadores pós-materialistas avaliam a importância da realização profissional, da política e do bemestar individual. Com base em um conjunto de investigações realizadas com esse instrumento, Inglehart (1991) mostra que as sociedades industriais podem ser classificadas em uma dimensão bipolar em função da importância que elas atribuem aos valores pós-materialistas e materialistas. Embora a validade dessa dimensão tenha sido contestada (Brechin \& Kempton, 1994), diversos estudos comparando culturas (Flanagan, 1987; Vala, 1993, 1994) e indivíduos (Bean \& Papadakis, 1994; Braithwaite, Makkai \& Pittelkow, 1996) revelam a existência de valores materialistas e pós-materialistas, organizados tanto em uma dimensão (Kidd \& Lee, 1997) quanto em duas dimensões correlacionadas positivamente (Marks, 1997; Van Deth, 1983).

As abordagens apresentadas reacendem o debate sobre a natureza dos valores. Eles são representantes das necessidades individuais, como defende Schwartz (1992), ou são indicadores sociais das mudanças culturais, como afirma Inglehart (1977)? Esse tipo de questionamento é central à natureza do objeto de estudo da Psicologia Social, dado que esta ciência tem-se desenvolvido em torno do debate sobre a relação indivíduosociedade (Pereira \& Soares, 2003). Esse debate também está implícito na pergunta sobre se os valores são construtos individuais ou sociais. Para tentar resolver esse problema, temse desenvolvido uma abordagem societal sobre os valores (Lima \& Camino, 1995; Pereira \& cols., 2001; Torres, 1992) que procura integrar os níveis individuais e sociais de análise dos valores. Essa abordagem considera tanto a perspectiva psicológica elaborada por Schwartz (1992) sobre os tipos motivacionais, quanto a perspectiva sociológica proposta por Inglehart (1991) sobre os 
valores materialistas e pós-materialistas. Nessa abordagem societal, os sistemas de valores são definidos como conhecimentos socialmente estruturados a partir dos diversos conteúdos ideológicos contidos na sociedade. Os valores são produções sociais e, portanto, precisam de um conjunto de condições sociais para sua emergência (Deschamps \& Devos, 1993). Essas condições são as lutas ideológicas travadas pelos grupos sociais em busca do poder (Pereira \& Camino, 1999). Neste sentido, a fonte dos valores encontra-se nas identidades ideológicas que orientam os grupos sociais (Da Costa, 2000).

Com base nesses pressupostos, tem-se realizado várias pesquisas com a finalidade de desenvolver um instrumento de medida, o Questionário de Valores Psicossociais ( $Q V P$ ), que permita fazer uma análise dos sistemas de valores de diversos grupos sociais. Inicialmente, Torres (1992) mostra que universitários paraibanos organizam em dois sistemas os valores que servem de base para uma sociedade ideal: democrático e autoritário. Com uma nova versão do instrumento, Lima e Camino (1995) identificam cinco sistemas subjacentes aos valores de estudantes de psicologia: bem-estar individual, bem-estar econômico, religioso, igualitário e libertário. Em outra investigação, Pereira, Lima e Camino (1997) confirmam a existência de cinco sistemas de valores em universitários paraibanos: bem-estar social; bem-estar individual; bem-estar profissional; religioso; bem-estar econômico.

Para identificar as dimensões psicossociais que organizam esses sistemas, Pereira e colaboradores (2001a) realizam duas investigações sobre os valores de universitários de João Pessoa. Com base nos resultados de uma Hierarchical Cluster Analysis (Aldenderfer \& Blashfild, 1984) e de uma Multidimensional Scaling (Kruskal \& Wish, 1978), Pereira e colaboradores verificam uma configuração composta por três sistemas: materialista, religioso e pós-materialista. O sistema pós-materialista é formado por três subsistemas: bem-estar social; bem-estar individual; bemestar profissional. Os coeficientes de fidedignidade dos sistemas são elevados nos dois estudos (alfas variando de 0,74 a 0,92 ). Para comparar a estrutura dos sistemas de valores de estudantes da Paraíba com a de estudantes da Região Sul do Brasil, Pereira e colaboradores (2001b) realizam duas novas investigações com amostras de universitários de Florianópolis e Porto Alegre. Nas duas cidades, a estrutura e o conteúdo dos sistemas são iguais às verificadas em João Pessoa. Os coeficientes de fidedignidade dos sistemas na Região Sul também são elevados (alfas variando entre 0,80 e 0,92, em Porto Alegre, e entre 0,79 e 0,92, em Florianópolis). Semelhanças conceituais entre o conteúdo do sistema materialista e os valores do poder (Schwartz, 1992) são destacadas, assim como entre o sistema pós-materialista e os valores de universalismo, benevolência e autodireção e entre o sistema religioso e os valores de conformidade e de tradição.

Em resumo, os estudos desenvolvidos com o QV P mostram os valores organizados em três sistemas: materialista; religioso; pós-materialista. Contudo, na lista de itens que formam esse instrumento não há um conjunto de valores hedonistas, identificados em diferentes culturas (Schwartz \& Bardi, 2001). Além disso, a abordagem societal dos valores estabelece uma associação conceitual entre os valores materialistas e os do poder social, entre os religiosos e os tipos motivacionais da tradição e conformidade, bem como entre os valores pós-materialistas e os do universalismo, da benevolência e da autodireção. Para averiguar se essas associações são empiricamente sustentáveis, este artigo apresenta um estudo que correlaciona a medida de valores obtida com o QVP com a medida de valores obtida com o SVS. O estudo é orientado por dois objetivos. Inicialmente, investiga a existência de um sistema de valores hedonistas através da inclusão de três valores ao QV P: sensualidade, sexualidade e uma vida excitante (Rokeach, 1973). Posteriormente, articula níveis de análises individuais e sociais dos valores através do estudo das associações entre sistemas de valores e tipos motivacionais. Espera-se que o sistema materialista se correlacione mais fortemente com os valores da autopromoção, particularmente com os do poder. Do mesmo modo, espera-se que o sistema religioso se correlacione mais fortemente com os valores de conservação, fundamentalmente com os tipos motivacionais da tradição e da conformidade. Espera-se também que o sistema pósmaterialista se correlacione mais fortemente com os valores da autotranscendência e com o tipo motivacional da autodireção (Pereira \& cols., 2001a).

\section{Método}

\section{Participantes}

Participaram nesta pesquisa uma amostra composta por 406 estudantes de ambos os sexos de cursos de Ciências Humanas de universidades localizadas na cidade de João Pessoa. A idade dos estudantes variou de 17 a 57 anos $(m=23,0 ; d p=5,62)$.

\section{Instrumentos}

Os dados foram coletados com um questionário contendo os seguintes instrumentos para mensurar os valores:

Questionário de Valores Psicossociais-QVP: como mencionado, ao QV P foram acrescentados três valores (sexualidade, sensualidade e uma vida excitante) com o objetivo de observar a formação de um sistema hedonista. Dessa forma, a escala fica composta pelos seguintes valores: alegria, amor, auto-realização, autoridade, competência, conforto, dedicação ao trabalho, fraternidade, igualdade, liberdade, lucro, justiça, realização profissional, responsabilidade, religiosidade, riqueza, salvação da alma, sensualidade, sexualidade, status, temor a Deus e uma vida excitante. Os participantes atribuíram uma nota variando entre um e cinco, considerando a importância de cada valor "para a construção de uma sociedade ideal" (o QVS encontra-se no Anexo A).

Schwartz. Values Survey - SVS: utilizou-se a versão do SVS validada no Brasil por Tamayo e Schwartz (1993), a qual contém 61 valores que avaliam 10 tipos motivacionais: poder, realização, hedonismo, estimulação, autodireção, universalismo, benevolência, tradição, conformidade e segurança. Os estudantes avaliaram cada valor como "um princípio guia na minha vida" utilizando uma escala variando de -1 (oposto aos meus valores) a 7 (de suprema importância). A análise da consistência interna dos tipos motivacionais apresenta os seguintes alfas: 0,44 para o poder (poder social, autoridade, riqueza, preservador da imagem, reconhecimento social e vaidade); 0,45 para 
realização (bem-sucedido, capaz, ambicioso, influente, inteligente, esperto); 0,64 para hedonismo (prazer e que goza a vida); 0,63 para estimulação (audacioso, uma vida variada e uma vida excitante); 0,48 para autodireção (curioso, criatividade, liberdade, escolhendo minhas metas, independente e auto-respeito); 0,70 para universalismo (protetor do ambiente, união com a natureza, um mundo de beleza, aberto, justiça social, sabedoria, um mundo em paz, igualdade, harmonia interior, sonhador); 0,73 para benevolência (prestativo, honesto, que perdoa, leal, responsável, uma vida espiritual, amizade verdadeira, amor maduro, sentido da vida e trabalho); 0,48 para tradição (ciente dos meus limites, devoto, humilde, respeito pela tradição, moderado e desprendimento); 0,59 para conformidade (obediente, respeitoso com os pais, autodisciplina e polidez); 0,62 para segurança (limpo, segurança nacional, reciprocidade de favores, ordem social, segurança familiar, senso de pertencer e saudável).

Esses coeficientes são similares aos verificados em estudos transculturais sobre o SVS (Schmitt, Schwartz, Steyer \& Schmitt, 1993; Schwartz, Verkasalo, Antonovsky \& Sagiv, 1997). De fato, a comparação dos coeficientes de consistência interna dos tipos motivacionais constatados neste estudo $(M=0,57)$ com os apresentados por Schwartz $(M=0,58)$ em suas publicações (Sagiv \& Schwartz, 1995, 2000; Schwartz, 1992) mostra que eles não se diferenciam significativamente $[t(40)=-0,09 ;$ n.s $]$. Com relação à cultura brasileira, esse tipo de comparação não é possível dado que os estudos que utilizam o SVS no Brasil não apresentam os coeficientes de fidedignidade dos tipos de valores (Tamayo, 1994; Tamayo \& Schwartz, 1993; Tamayo \& cols., 1996; Tamayo, Lima, Marques \& Martins, 2001).

\section{Procedimentos}

Os estudantes responderam os questionários em salas de aulas definidas através de sorteios. Para a realização dos sorteios, solicitou- se a grade curricular aos coordenadores dos cursos. Foram sorteadas disciplinas de início, meio e final de curso. Em seguida, solicitou-se a permissão aos professores das disciplinas para as aplicações dos questionários. Os estudantes utilizaram um intervalo de tempo que variou de 30 a 40 minutos para responder todos os itens dos instrumentos.

\section{Resultados e Discussões}

Para avaliar a forma como os estudantes configuram os diversos valores do QVP e as dimensões subjacentes aos sistemas, foram aplicadas as duas técnicas estatísticas empregadas nos estudos desenvolvidos por Pereira e colaboradores (2001a): a HCA e a MDS. A HCA utiliza medidas de dissimilaridades (distâncias euclidianas) entre as variáveis para a construção de grupos ou clusters de variáveis (Everitt, 1980). Como método de classificação das variáveis usou-se o desenvolvido por Ward (1963). Na MDS, as distâncias euclidianas produzem um tipo de mapa psicossocial que permite acessar dimensões subjacentes à adesão feita pelos estudantes aos valores. $\mathrm{O}$ grau de adequação do número de dimensões necessárias para explicar essa adesão foi medido pelo coeficiente de Stress, o qual deve ser inferior a 0,15 para uma análise adequada dos resultados obtidos (Abelson, 1967).

Como esperado, os resultados da HCA mostram a formação de quatro agrupamentos (clusters) de valores. Os valores status, riqueza, lucro e autoridade constituem o sistema de valores materialistas. Os valores uma vida excitante, sensualidade, prazer e sexualidade formam o sistema hedonista. Verifica-se também um conjunto homogêneo de valores, chamado de pós-materialista, que é constituído pelos valores do bem-estar social (igualdade, liberdade, fraternidade e justiça), do bem-estar individual (conforto, auto-realização, alegria e amor) e por valores do trabalho (realização profissional, dedicação

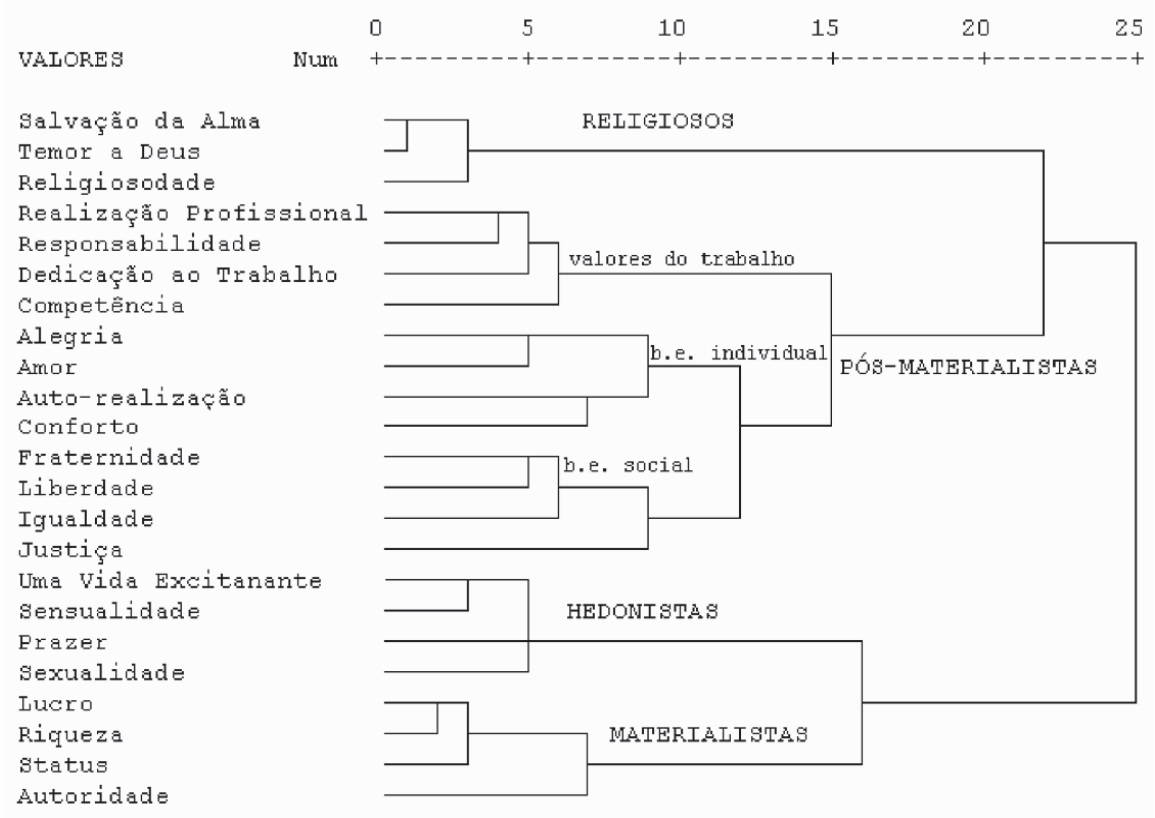

Figura 1. Dendrograma representando a estrutura dos sistemas de valores. 
ao trabalho, competência e responsabilidade). Os valores temor a Deus, religiosidade e salvação da alma formam o sistema de valores religiosos.

Os resultados da $M D S$ mostram (Figura 2) esses sistemas distribuídos em função de duas dimensões (Stress=0,07 e $\left.\mathrm{R}^{2}=0,98\right)$. Na primeira dimensão, reaparece a distinção proposta por Inglehart (1977) e por Pereira e colaboradores (2001a) entre valores materialistas e pós-materialistas. A segunda dimensão contrapõe o sistema religioso ao sistema hedonista. Com base nos resultados da $H C A$ e da $M D S$, pode-se considerar que os estudantes organizam seus valores com base em quatro sistemas: o materialista, o religioso, o hedonista e o pós-materialista, os quais mostram alfas iguais a $0,74,0,80,0,74$ e 0,75, respectivamente, na análise da consistência interna desses sistemas.

No conjunto, os resultados apresentados corroboram as hipóteses formuladas (Pereira \& cols., 2001a). A inclusão de três valores ao QV P permite que os estudantes organizem sua adesão a esses valores a partir de um sistema hedonista, tal como verificado em diversas investigações transculturais (Schwartz, 1992, 1994). Além disso, esse sistema hedonista é configurado em oposição aos valores religiosos na projeção dos resultados da $M D S$, assim como o sistema materialista em contraposição ao pós-materialista. De fato, considerando o sistema religioso conceitualmente semelhante aos valores de conservação, não é de se estranhar uma contraposição entre esse sistema e os valores hedonistas, pois esses sistemas podem representar interesses antagônicos (Schwartz, 1996). Do mesmo modo, seguindo as suposições de Inglehart (1991), é provável uma oposição entre materialismo e pós-materialismo, dado que os valores que compõem esses sistemas emergiram em conjunturas sócioeconômicas diferenciadas. Dada a natureza dos valores que se tem explicitado neste trabalho, essas interpretações devem ser ponderadas, como mostram as correlações positivas entre os sistemas de valores e os tipos motivacionais (Tabela 1).

As correlações entre os sistemas de valores do QVP e os tipos motivacionais da teoria de Schwartz (1992) são positivas e estatisticamente significantes. Como esperado, o sistema materialista se correlaciona com os valores do poder social $(r=0,57 ; p<0,001)$. O sistema pós-materialista se correlaciona mais fortemente com os valores do universalismo $(r=0,55$; $p<0,001)$ e da benevolência $(r=0,53 ; p<0,001)$. Os valores da autodireção, da conformidade e da segurança correlacionamse mais fortemente com o sistema pós-materialista $(r=0,40$; $p<0,001 ; \quad r=0,42 ; \quad p<0,001 ; \quad r=0,45 ; \quad p<0,001$, respectivamente). Os tipos motivacionais do hedonismo e da estimulação correlacionam-se com o sistema hedonista avaliado com o QVP $(r=0,50 ; p<0,001 ; r=0,45 ; p<0,001$, respectivamente). O sistema religioso se correlaciona com os valores da tradição $(r=0,51 ; p<0,001)$, da benevolência $(r=0,40 ; p<0,001)$ e da conformidade $(r=0,40 ; p<0,001)$.

As correlações entre os sistemas de valores do QVP e os tipos motivacionais de ordem superior são mais elucidativas (Tabela 2). O sistema materialista correlaciona-se com os valores da autopromoção $(r=0,49 ; p<0,001)$, o pós-materialista com os

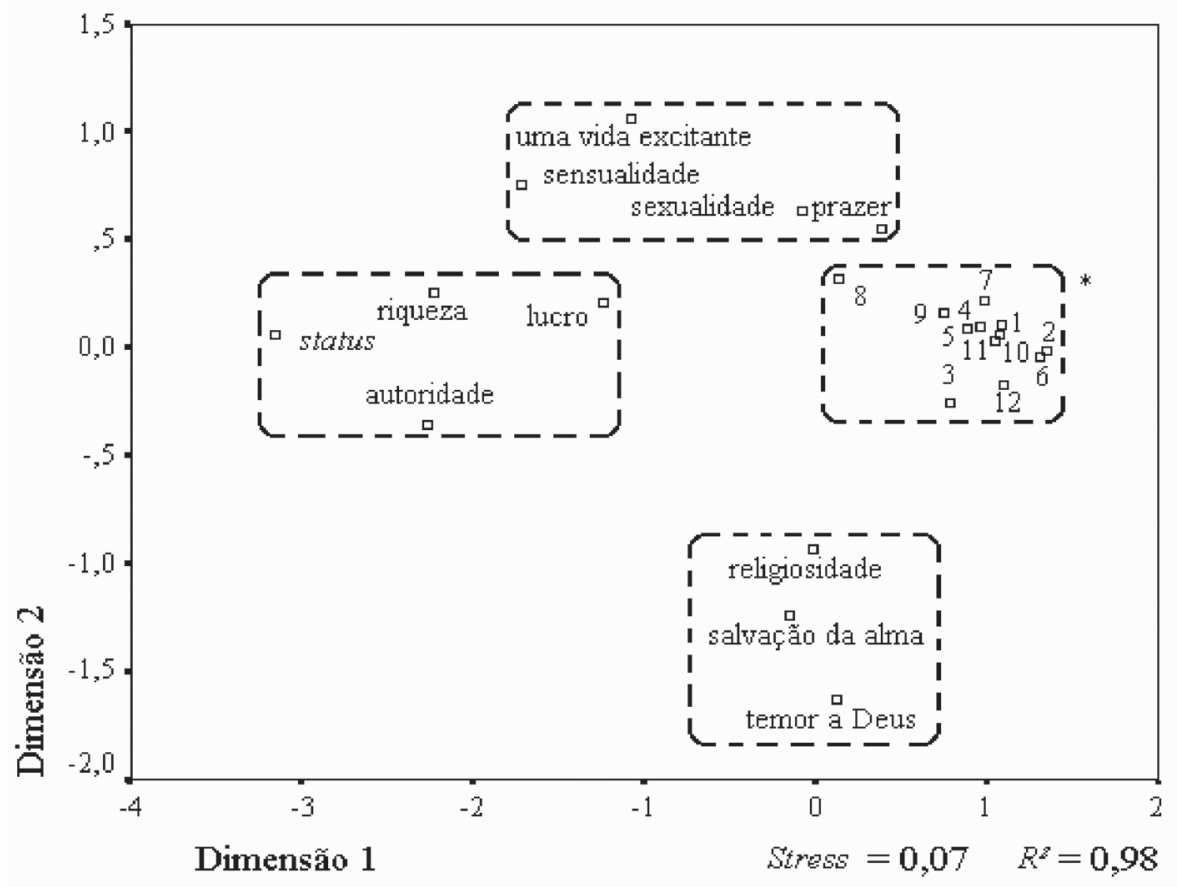

Figura 2. Representação gráfica dos resultados da multidimensional scaling aplicada aos valores.

Nota.* Os valores contidos neste quadro são os seguintes: $1=$ igualdade; $2=$ justiça; $3=$ liberdade; $4=$ fraternidade; $5=$ alegria; $6=$ auto-realização; 7=amor; $8=$ conforto; $9=$ competência; $10=$ dedicação ao trabalho; $11=$ realização profissional; $12=$ responsabilidade. 
Tabela 1

\begin{tabular}{|c|c|c|c|c|}
\hline \multirow[b]{2}{*}{ Tipos Motivacionais } & \multicolumn{3}{|c|}{ Sistemas de Valores do QVP } & \multirow[b]{2}{*}{ Pós-Materialista } \\
\hline & Materialista & Religioso & Hedonista & \\
\hline Poder/social & 0,57 & & & \\
\hline Realização & & & & \\
\hline Hedonismo & & & 0,50 & \\
\hline Estimulação & & & 0,45 & \\
\hline Auto-direção & & & & 0,40 \\
\hline Universalismo & & & & 0,55 \\
\hline Benevolência & & 0,40 & & 0,53 \\
\hline Tradição & & 0,51 & & 0,46 \\
\hline Conformidade & & 0,40 & & 0,42 \\
\hline Segurança & & & & 0,45 \\
\hline
\end{tabular}

Nota. Apenas as correlações iguais ou superiores a 0,40 são apresentadas. Todas as correlações apresentadas são estatisticamente significantes $\operatorname{com} p<0,001$.

Tabela 2

Coeficientes de Correlações ( $r$ de Pearson) entre os Sistemas de Valores do QV P e os Tipos Motivacionais de Segunda Ordem Avaliados com o SVS

\begin{tabular}{|c|c|c|c|c|}
\hline \multirow[b]{2}{*}{ Tipos Motivacionais } & \multicolumn{3}{|c|}{ Sistemas de Valores do QVP } & \multirow[b]{2}{*}{ Pós-Materialista } \\
\hline & Materialista & Religioso & Hedonista & \\
\hline Auto-promoção & 0,49 & & & \\
\hline Conservação & & 0,52 & & 0,50 \\
\hline Abertura à mudança & & & 0,55 & \\
\hline Autotranscendência & & & & 0,63 \\
\hline
\end{tabular}

Nota. Apenas as correlações iguais ou superiores a 0,40 são apresentadas. Todas as correlações apresentadas são estatisticamente significantes $\operatorname{com} p<0,001$.

valores da autotranscendência $(r=0,63 ; p<0,001)$, o religioso com os valores de conservação $(r=0,51 ; p<0,001)$ e o hedonista com os valores de abertura à mudança $(r=0,55 ; p<0,001)$. Os valores de conservação também apresentam correlação elevada com o sistema pós-materialista $(r=0,50 ; p<0,001)$. Este conjunto de correlações corrobora a aproximação conceitual feita por Pereira e colaboradores (2001a) entre os sistemas de valores do QVP e os tipos motivacionais do SVS.

Embora os resultados da MDS localizem os sistemas em lados opostos na projeção bidimensional, eles podem se dever ao que tem sido definido nos estudos sobre os valores como artefato metodológico (Lima, 1997; Vala, 1993). Esse artefato pode ser confirmado na medida em que as intercorrelações entre os sistemas de valores do QV P e os tipos motivacionais do SVS são positivamente significantes. Resultados similares a estes também são constatados nos estudos dos valores de universitários de João Pessoa, de Florianópolis e de Porto Alegre (Pereira \& cols, 2001a, 2001b). As correlações positivas parecem corroborar o raciocínio de que não existe oposição motivacional entre valores individuais e coletivos. Eles representam as experiências de diferentes grupos sociais e se formam no interior desses através do consenso, da comparação social e da pluralidade de opiniões e de crenças sobre a realidade social (Deschamps \& Devos, 1993; Vala, 1994). Neste sentido, a contraposição verificada entre os sistemas de valores é um artefato metodológico difícil de ser verificado na arena sócio-político contemporânea (Lima, 1997). De fato, os sistemas de valores, sendo considerados como estruturas de conhecimento derivadas das ideologias construídas no interior dos grupos sociais, não podem apresentar relações de oposição motivacional.

\section{Conclusões}

Este artigo apresenta uma abordagem societal aplicável à análise dos sistemas de valores de estudantes universitários. Inicialmente, são apresentadas as principais teorias, em Psicologia Social, desenvolvidas sobre os valores, identificando como essas teorias concebem a natureza e a fonte dos valores, bem como os procedimentos metodológicos empregados por cada uma delas no estudo dos valores sociais. Sobretudo, tenta-se compreender, com base nos níveis de explicações elaborados pela Psicologia Social para os fenômenos sociais (Doise, 1982), os elementos fundamentais dos valores destacados por essas teorias, propondo-se, como sugerido por Doise (1976), a articulação dos níveis de análises para a compreensão da natureza dos sistemas de valores. Como salientado (Torres \& cols., 2001), tanto a abordagem de Rokeach (1973) quanto a de Schwartz (1992) enfatizam a natureza individual dos valores ao defini-los como crenças ou concepções individuais decorrentes 
das necessidades humanas. É nesse sentido que a perspectiva adotada por esses autores também pode ser considerada um exemplo de explicações intraindividuais, tal como as destacadas na análise feita por Doise (1982) das diversas teorias em Psicologia Social. Contudo, tanto neste artigo quanto em outras investigações (Pereira \& cols. 2001a, 2001b) têm-se apontado algumas limitações nessas explicações dado que elas, aos vincular os valores às necessidades ou às motivações individuais, parecem distancia-los do seu aspecto mais central - a sua natureza social. Essa limitação torna relevante a análise de outra abordagem sobre valores, desenvolvida por um sociólogo (Inglehart, 1977), que embora conceba os valores não como crenças individuais, mas como indicadores sociológicos das mudanças culturais decorrentes das transformações econômicas, ainda os vinculam, paradoxalmente, a uma hierarquia de necessidades (Inglehart, 1991).

Com base nessas insatisfações, este artigo propõe uma perspectiva de análise dos valores, também explicitada em outros trabalhos (Da Costa, 200; Lima, 1997; Pereira \& cols., 2001a), que pretende dar uma explicação societal tanto para a natureza dos valores quanto para suas fontes. Esta perspectiva se diferencia das outras abordagens em três aspectos fundamentais. Com relação ao primeiro aspecto, enquanto Rokeach (1973) e Schwartz (1992) enfatizam a natureza individual dos valores e Inglehart (1991) destaca sua dimensão sociológica, a abordagem adotada neste trabalho prioriza fatores societais na natureza dos valores ao defini-los como estruturas sociais de conhecimentos que são elaboradas no interior dos grupos sociais. Com relação às fontes dos sistemas de valores, enquanto Rokeach (1973), Schwartz (1992) e Inglehart (1991) atribuem, como fonte dos valores, um conjunto de necessidades individuais, este artigo destaca as ideologias vigentes nas sociedades como sendo as responsáveis pelo desenvolvimento desses valores no interior dos grupos sociais (Torres \& cols., 2001). Com relação ao terceiro aspecto em que as abordagens se distanciam, os procedimentos metodológicos para o estudo dos valores, enquanto na perspectiva psicológica solicita-se aos sujeitos que avaliem os valores como "princípios guia em minha vida" e na sociológica como "metas a serem atingidas pelo país", na abordagem societal, considerando os valores como decorrentes das ideologias existentes sobre a natureza da sociedade, solicita-se aos membros dos diversos grupos sociais que atribuam notas aos valores "considerando a sua importância para a construção de uma sociedade ideal". É nesse sentido que essa abordagem, ao situar os valores nas relações ideológicas, também pode ser considerada exemplar dos estudos classificados por Doise (1982) no quarto nível de explicação em psicologia social.

É com base nesta perspectiva que o estudo apresentado analisa a configuração dos sistemas de valores de estudantes universitários paraibanos. Os resultados da HCA e da $M D S$ mostram que, para os universitários de João Pessoa, os valores se estruturam em quatro sistemas: o hedonista, o religioso, o materialista e o pós-materialista. O sistema hedonista reúne os valores ligados ao prazer e à satisfação sexual (prazer, sexualidade, sensualidade e uma vida excitante). O sistema religioso se caracteriza por integrar os valores típicos da espiritualidade cristã dominante na cultura brasileira: temor a Deus, religiosidade e salvação da alma. O sistema materialista organiza valores ligados ao bem-estar econômico (riqueza e lucro) e à estratificação social (status e autoridade) como objetivos a conseguir numa sociedade ideal. Finalmente, o sistema pós-materialista integra, como proposto por Inglehart (1991) e por Pereira e colaboradores (2001), um conjunto de subsistemas de valores que vão de valores do bem-estar social (igualdade, liberdade, fraternidade e justiça) ao bem-estar individual (conforto, auto-realização, alegria e amor), passando pelos valores concernentes ao trabalho e ao bem-estar profissional (realização profissional, dedicação ao trabalho, competência e responsabilidade).

No que se refere ao conteúdo dos sistemas, está estabelecida a convergência entre os sistemas obtidos com o QVP (Pereira \& cols., 2001a) e os tipos motivacionais da teoria de Schwartz (1992). Como esperado, o sistema religioso relaciona-se com o tipo motivacional da conservação (fundamentalmente, os da tradição e conformidade), pois ambos traduzem um estilo de vida estruturado em torno da harmonia social e da manutenção do statu quo (Schwartz, 1994). O sistema materialista assemelha-se ao tipo motivacional da autopromoção, fundamentalmente aos valores que descrevem o poder social. O conteúdo do sistema pós-materialista assemelha-se mais fortemente aos valores do universalismo e da benevolência, ambos descritores da autotranscendência. Estes resultados corroboram dados de várias pesquisas em que valores pós-materialistas associam-se aos tipos motivacionais do universalismo e da benevolência enquanto valores materialistas descrevem o tipo motivacional da segurança (Helkama, Uutela \& Schwartz, 1992) e do poder (Pereira \& Camino, 1999). Além disso, Braithwaite (1994) encontra associações entre materialismo e os valores da ordem social e da segurança nacional. Finalmente, o conteúdo do sistema hedonista apresenta associações empíricas com os valores da abertura à mudança.

O conjunto dos resultados mostra não apenas a validade de construto do QVP, mas a análise das correlações entre oQVP e o SVS indica a existência de um aspecto peculiar aos valores, expresso na unanimidade de correlações positivamente constatada entre os sistemas e os tipos motivacionais. Uma análise suplementar das correlações que não foram apresentadas nas Tabelas 1 e 2 porque são inferiores a 0,40 confirmam essa afirmação. Essa análise revela que não há correlações negativas entre os valores do QVP e do SVS. Além disso, os sistemas de valores do QVP, assim como os tipos motivacionais do SVS não se correlacionam negativamente. As correlações positivas mostram que uma concepção baseada em relações de conflito entre valores (Schwartz, 1996) contradiz a existência da desejabilidade social dos sistemas axiológicos. Isto não implica que não possa haver conflitos entre motivações individuais e sociais. Significa que os valores não são representantes cognitivos de interesses ou motivações individuais. Deste modo, a cisão entre valores individuais e sociais é insustentável. Todos os valores são sociais na medida em que são produzidos nas interações entre 
os homens (Beattie, 1980; Lima, 1997) e são amplamente compartilhados por estes (Maio \& Olson, 1998). Assim, ao responderem questionários de valores, os participantes das pesquisas acessam os conhecimentos socialmente estruturados a partir dos conteúdos ideológicos que organizam a sociedade (Da Costa, 2000). Esse processo ocorre independente das instruções solicitarem respostas baseadas nos valores como guia na vida das pessoas, metas a serem atingidas pelo país ou como sendo importantes para uma sociedade ideal. É basicamente este o significado das correlações positivas entre os valores medidos com o SVS e com o QVP. Entretanto, para que essa afirmação seja mais robusta empiricamente, é necessária a realização de novas investigações que analisem correlações entre dados obtidos com esses dois instrumentos e a escala de valores apresentada por Inglehart (1991). Outro procedimento viável para testar essas idéias é a manipulação experimental das instruções utilizando um dos instrumentos.

Considerar os valores como construtos sociais e não individuais permite compreender uma aparente ambigüidade no mundo pósmoderno. Trata-se da ampla importância atribuída às aspirações moralistas de fraternidade e igualdade juntamente com preocupações relacionada à aplicação concreta da justiça num mundo em que a ideologia dominante descreve o espírito competitivo como sendo a essência da natureza humana (Billig, 1991; Camino, Silva, Machado \& Pereira, 2001). Paradoxalmente, o mundo moderno enfatiza tanto a centralidade dos valores pós-materialistas para o bom desenvolvimento da sociedade quanto, cada vez mais, subordina-se às leis do mercado globalizado, visando lucro e acúmulo econômico característicos do materialismo. É, portanto, a ideologia política, que por natureza é contraditória (Camino, 1996; Camino, Lima \& Torres, 1997), que talvez explique as correlações positivas entre os sistemas de valores. Apenas novas investigações envolvendo os sistemas de valores e as ideologias políticas poderão fundamentar este raciocínio.

\section{Referências}

Abelson, R. P. (1967). A technique and a model of multi-dimensional attitude scaling. Em M. Fishbein (Org.), Readings in attitude: Theory and measurement (pp. 349-356). New York: Jonh Wiley \& Sons.

Aldenderfer, M. S. \& Blashfield, R. K. (1984). Cluster analysis. Beverly Hills: Sage.

Bean, C. \& Papadakis, E. (1994). Polarized priorities or flexible alternatives? Dimensionality in Inglehart's materialism-posmaterialism scale. International Journal of Public Opinion Research, 6, 264-297.

Beattie, J. (1980). Introdução à antropologia social. São Paulo: Companhia Editora Nacional.

Billig, M. (1991). Ideology and opinions: Studies in rhetorical psychology. London: Sage.

Bond, M. H. (1988). Finding universal dimensions of individual variation in multicultural studies of values: The Rokeach and Chinese value survey. Journal of Personality and Social Psychology, 49, 250-264.

Braithwaite, V. (1994). Beyond Rokeach's equality-freedom model: Two-dimensional values in a one-dimensional world. Journal of Social Issues, 50, 67-94.

Braithwaite, V. \& Law, H. G. (1985). Structure of human values: Testing the adequacy of the Rokeach Value Survey. Journal of Personality and Social Psychology, 49, 250-263.

Braithwaite, V. \& Scott, W. (1991). Values. Em J. P. Robinson, P. R. Shaver \& L. S. Wrightsman (Orgs.), Measures of personality and social psychological attitudes (pp. 661-753). San Diego: Academic Press.
Braithwaite, V., Makkai, T.\& Pittelkow, Y. (1996). Inglehart's materialism-postmaterialism concept: Clarifying the dimensionality debate through Rokeach's model of social values. Journal of Applied Social Psycbology, 26, 1536-1555.

Brechin, S. R. \& Kempton, W. (1994). Global environmentalism: A challenge to the postmaterialism thesis? Social Science Quarterly, 75, 245-269.

Camino, L. (1996). Uma abordagem psicossociológica no estudo do comportamento político. Psicologia e Sociedade, 8, 16-42.

Camino, L., Lima, M. \& Torres, A. (1997). Ideologia e espaço político em estudantes universitários. Em L. Camino, L. Lhullier \& S. Sandoval (Orgs.), Estudos sobre comportamento político (pp. 87-105). Florianópolis: Letras Contemporâneas.

Camino, L., Silva, P., Machado, A. \& Pereira, C. (2001). A face oculta do racismo no Brasil: Uma análise psicossociológica. Revista de Psicologia Politica, 1, 13-36.

Canter, D. (1985). Facet theory: Approaches to social research. New York: SpringerVerlag.

Chinese Culture Connection (1987). Chinese values and the search for culture-free dimensions of culture. Journal of Cross-Cultural Psychology, 18, 143-164.

$\mathrm{Da}$ Costa, J. B. (2000). Visões sociais de democracia: Um estudo psicosssociológico dos significados da democracia. Tese de Doutorado não-publicada, Curso de Pós-graduação em Psicologia Social, Pontifícia Universidade Católica de São Paulo. São Paulo, SP.

Davison, M. (1983). Multidimensional scaling. New York: Wiley.

Deschamps, J. \& Devos, T. (1993). Valeurs, cultures et changement. Intercultures, 1, $17-28$.

Doise, W. (1976). L'articulation psychosociologique et les relations entre groupes. Bruxelas: De Boeck.

Doise, W. (1982). L'explicacation en psychologie sociale. Paris: PUF.

Everitt, B. (1980). Cluster analysis. New York: Halsted.

Feather, N. T. (1990). Bridging the gap between values and actions: Recent applications of the expectancy-value model. Em E. T. Higgins \& R. M. Sorrentino (Org.), The handbook of motivation and cognition: Foundations of social behavior (Vol. 2; pp. 151-92). New York: Guilford Press.

Flanagan, S. C. (1987). Value change in industrial societies. American Political Science Revien, 81, 1303-1319.

Guttman, L. (1968). A general no metric technique for finding the smallest coordinate space for a configuration of points. Psychometrica, 33, 469-506.

Helkama, K., Uutela, A. \& Schwartz, S. (1992). Value systems and political cognition. Em G. M. Breakwell (Org.), Social psychology of political and economic cognition (pp. 7-31). London: Surrey University.

Inglehart, R. (1977). The silent revolution. Princeton: University Press.

Inglehart, R. (1991). El cambio cultural en las sociedades industriales avanzadas. Madrid: Siglo XXI.

Inglehart, R. (1994). Modernización y post-modernización: La cambiante relación entre el desarrollo económico, cambio cultural y político. Em J. D. Nícolas \& R. Inglehart (Orgs.), Tendencias mundiales de cambio en los valores sociales y politicos (pp. 157-170). Madrid: Fundesco.

Kidd, Q. \& Lee, A. R. (1997). Postmaterialist values and the environment: A critique and reappraisal. Social Science Quarterly, 78, 1-15.

Kluckhohn, C. (1968). Los valores y las orientaciones de valor en la teoría de la acción. Em T. Parsons \& E. A. Shils (Orgs.), Hacia una teoría general de la acción (pp. 435-485). Buenos Aires: Editorial Kapelusz.

Kruskal, J. B. \& Wish, M. (1978). Multidimensional scaling. London: Sage.

Lima, M. E. (1997). Valores, participação política, atitudes face a democracia e ao autoritarismo: Uma análise da socialiração politica dos universitários da Paraíba. Dissertação de Mestrado não-publicada, Universidade Federal da Paraíba. João Pessoa, PB.

Lima, M. E. \& Camino, L. (1995). A política na vida de estudantes universitários: Uma análise em termos de espaço político e de valores. Em M. J. L. Silva (Org.), Iniciados (pp. 11-35). João Pessoa: Editora Universitária.

Maio, G. R. \& Olson, J. M. (1998). Values as truisms: Evidence and implications. Journal of Personality and Social Psychology, 74, 294-311.

Maio, G. R., Olson, J. M., Allen, L. \& Bernard, M. (2001). Addressing discrepancies between values and behavior: The motivating effect of reasons. Journal of Experimental Social Psychology, 37, 104-117.

Marks, G. N. (1997). The formation of materialist and pos-materialist values. Social Science Research, 26, 52-68.

Maslow, A. K. (1954). Motivation and personality. New York: Harper \& Row.

Menezes, I. \& Campos, B. (1997). The process of value-meaning construction: A cross-sectional study. European Journal of Social Psychology, 27, 55-77.

Pereira, C. \& Camino, L. (1999). Proposta de um modelo psicossociológico para o estudo das atitudes políticas de estudantes universitários: Uma análise em termos de valores materialistas e pós-materialistas. Em M. F. V. Souza (Org.), Iniciados (pp. 427-440). João Pessoa: Editora Universitária. 
Pereira, C. \& Soares, A. (2003). Reflexões acerca da perspectiva das representações sociais. Estudos, 30, 61-84.

Pereira, C., Camino, L., Da Costa, J., Lima, M. E., Lhullier, L. \& Sandoval, S. (2001b). Sistemas de valores e atitudes democráticas de estudantes universitários do Sul do Brasil. Estudos, 28, 639-671.

Pereira, C., Lima, M. E. \& Camino, L. (1997). A prática política: Uma análise psicossociológica em termos de valores e inserção social. Em M. J. L. Silva (Org.), Iniciados (pp. 199-235). João Pessoa: Editora Universitária.

Pereira, C., Lima, M. E. \& Camino, L. (2001a). Sistemas de valores e atitudes democráticas de estudantes universitários de João Pessoa. Psicologia: Reflexão e Crítica, 14, 177-190.

Rokeach, M. (1968). Beliefs, attitudes and values: A theory of organization and change. São Francisco: Jossey-Bass.

Rokeach, M. (1973). The nature of buman values. New York: Free Press.

Rokeach, M. (1979a). Introduction. Em M. Rokeach (Org.), Understanding buman values: Individual and societal (pp. 1-11). New York: Free Press.

Rokeach, M. (1979b). The two-value model of political ideology and British politics. Em M. Rokeach (Org.), Understanding human values: Individual and societal (pp. 192-196). New York: Free Press.

Sagiv, L. \& Schwartz, S. H. (1995). Value priorities and readiness for out-group social contact. Journal of Personality and Social Psychology, 69, 437-448.

Sagiv, L. \& Schwartz, S. H. (2000). Value priorities and subjective well-being: Direct relations and congruity effects. European Journal of Social Psychology, 30, $177-$ 198.

Schmitt, M. J., Schwartz, S. H., Steyer, R. \& Schmitt, T. (1993). Measurement models for the Schwartz Value Inventory. European Journal of Psychology Assessment, 9, 107-121.

Schwartz, S. H. (1992). Universals in the content and structure of values: Theoretical advanced and empirical testes in 20 countries. Em M. Zanna (Org.), Advanced in experimental social psychology (Vol. 25; pp. 1-65). Orlando: Academic Press.

Schwartz, S. H. (1994). Are there universal aspects in the structure and contents of human values? Journal of Social Issues, 50, 19-45.

Schwartz, S. H. (1996). Value priorities and behavior: Applying a theory of integrated value systems. Em C. Seligman, J. M. Olson \& M. P. Zanna (Orgs.), The psychology of values: The Ontario Symposium (Vol. 8; pp. 1-24). Mahwah, NJ: LEA.

Schwartz, S. H. (1999). A theory of cultural values and some implications for work. Applied Psychology: An International Review, 48, 23-47.

Schwartz, S. H. \& Bardi, A. (2001). Value hierarchies across cultures: Taking a similarities perspective. Journal os Cross-Cultural Psychology, 32, 268-290.

Schwartz, S. H. \& Bilsky, W. (1987). Toward a universal psychological structure of human values. Journal of Personality and Social Psychology, 53, 550-562.
Schwartz, S. H. \& Bilsky, W. (1990). Toward a theory of the universal content structure of values: Extensions and cross-cultural replications. Journal of Personality and Social Psychology, 58, 878-891.

Schwartz, S. H., Verkasalo, M., Antonovsky, A. \& Sagiv, L. (1997). Value priorities and social desirability: Much substance, some style. British Journal of Social Psychology, 36, 3-18.

Seligman, C. \& Katz, A. (1996). The dynamics of value systems. Em C. Seligman, J. M. Olson \& M. P. Zanna (Orgs.), The psychology of values: The Ontario Symposium (Vol. 8; pp. 53-75). Mahwah, NJ: LEA.

Tamayo, A. (1994). Hierarquia de valores transculturais e brasileiros. Psicologia: Teoria e Pesquisa, 10, 269-285.

Tamayo, A. \& Shwartz, S. H. (1993). Estrutura motivacional dos valores humanos. Psicologia: Teoria e Pesqusa, 9, 328-346.

Tamayo, A., Lima, A., Marques, J. \& Martins, L. (2001). Prioridades axiológicas e uso de preservativo. Psicologia: Reflexão e Crítica, 14, 167-175.

Tamayo, A., Pimenta, M., Rolim, M., Rodovalho, O. \& Castro, P. (1996). Prioridades axiológicas e orientação política. Psicologia: Teoria e Pesquisa, 12, 253-259.

Torres, A. R. R. (1992). Uma análise psicossocial da identificação partidária: $O$ caso dos estudantes da UFPb nas eleições de 1988, 1989 e 1990. Dissertação de Mestrado não-publicada, Universidade Federal da Paraíba. João Pessoa, PB.

Torres, A. R. R., Pereira, C., Guimarães, J., Albernaz, M., Vieira, S. \& Barros, T. (2001). Valores e psicologia social: Modelos de análise. Estudos, 28, 539-559.

Vala, J. (1993). Valores sócio-políticos. Em L. de França (Org.), Portugal, valores europeus e identidade cultural (pp. 221-259). Lisboa: Instituto de Estudos para o Desenvolvimento.

Vala, J. (1994). La emergencia de los valores post-materialistas en Portugal. Em J. D. Nícolas \& R. Inglehart (Orgs.), Tendencias mundiales de cambio en los valores sociales ypoliticos (pp. 157-170). Madrid: Fundesco.

Van Deth, J. W. (1983). Ranking the ratings: The case of materialist and posmaterialist value orientations. Political Methodology, 9, 407-431.

Ward, J. H. (1963). Hierarchical grouping to optimize an objective function. Journal of American Association, 58, 236-244.

Weber, M. (1904/1994). A ética protestante e o espírito do capitalismo. São Paulo: Pioneira.

Williams, R. M. (1979). Change and stability in values and value systems: A sociological perspective. Em M. Rokeach (Org.), Understanding buman values: Individual and societal (pp. 15-46). New York: Free Press.

Recebido: 14/05/2003

Última Revisão: 28/01/2004

Aceite Final:04/03/2004

Sobre os autores

Cícero Pereira é Professor de Psicologia Social na Universidade Católica de Goiás. Desenvolve pesquisas sobre Valores, Comportamento Político, Direitos Humanos e Processos de Exclusão Social.

Leoncio Camino é Professor no Programa de Pós-Graduação em Psicologia Social da Universidade Federal da Paraíba. É membro da Comissão de Direitos Humanos do Conselho Federal de Psicologia e desenvolve pesquisas sobre Direitos Humanos, Processos de Exclusão Social e Comportamento Político.

Joseli Bastos da Costa é Professor no Programa de Pós-Graduação em Psicologia Social da Universidade Federal da Paraíba. É coordenador do Grupo de Pesquisa em Comportamento Político da UFPB. Desenvolve pesquisas sobre Valores e Comportamento Político. 


\section{Anexo A}

\section{Questionário de Valores Psicossociais (QVP)}

\section{Instruções}

Logo abaixo você encontrará uma lista contendo um conjunto de valores sociais aos quais você deverá atribuir uma nota variando de 1 (um) a 5 (cinco), considerando o grau de importância de cada um dos valores para a construção de uma sociedade ideal para se viver. Lembre-se que quanto menor for a nota, menor será a importância do valor e, quanto maior for a nota, maior será a importância do valor.

\begin{tabular}{|c|c|c|}
\hline & $\underline{\text { Nota }}$ & \\
\hline 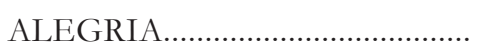 & [ ] & AMOR \\
\hline AUTO-REALIZAÇÃO................... & [ ] & 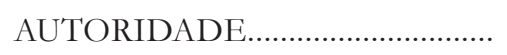 \\
\hline 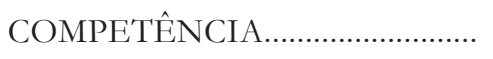 & {$[\quad]$} & 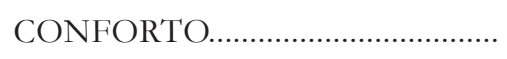 \\
\hline DEDICAÇÃO AO TRABALHO. & {$\left[\begin{array}{ll}1 & \end{array}\right.$} & 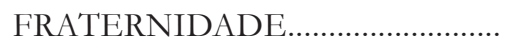 \\
\hline IGUALDADE & & 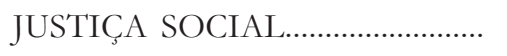 \\
\hline 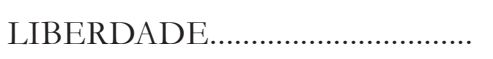 & [ ] & 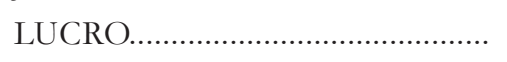 \\
\hline 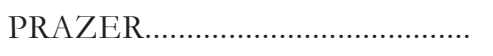 & {$\left[\begin{array}{ll}1 & \end{array}\right.$} & REALIZAÇÃO PROFISSIONAL. \\
\hline 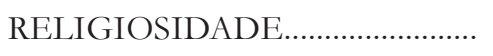 & {$\left[\begin{array}{l}1 \\
{[}\end{array}\right.$} & RESPONSABILIDADE....................... \\
\hline RIQUEZA & {$\left[\begin{array}{ll}1 & 0\end{array}\right.$} & SALVAÇÃO DA ALMA.................. \\
\hline SENSUALIDADE $\ldots \ldots \ldots \ldots . . . . . . . . . . . . . . .$. & {$\left[\begin{array}{ll}1 & 0\end{array}\right.$} & SEXUALIDADE \\
\hline 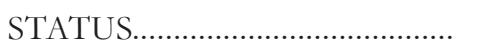 & [ ] & 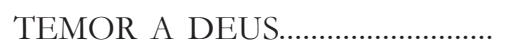 \\
\hline
\end{tabular}

